\title{
Review
}

\section{Testing evolutionary hypotheses about human biological adaptation using cross-cultural comparison}

\author{
Ruth Mace*, Fiona Jordan, Clare Holden \\ Department of Anthropology, University College London, Gower St, London WC1E 6BT, UK
}

Received 4 July 2002; received in revised form 16 January 2003; accepted 17 January 2003

\begin{abstract}
Physiological data from a range of human populations living in different environments can provide valuable information for testing evolutionary hypotheses about human adaptation. By taking into account the effects of population history, phylogenetic comparative methods can help us determine whether variation results from selection due to particular environmental variables. These selective forces could even be due to cultural traits-which means that geneculture co-evolution may be occurring. In this paper, we outline two examples of the use of these approaches to test adaptive hypotheses that explain global variation in two physiological traits: the first is lactose digestion capacity in adults, and the second is population sex-ratio at birth. We show that lower than average sex ratio at birth is associated with high fertility, and argue that global variation in sex ratio at birth has evolved as a response to the high physiological costs of producing boys in high fertility populations.
\end{abstract}

(C) 2003 Elsevier Science Inc. All rights reserved.

Keywords: Comparative method; Human sex ratio; Lactose tolerance; Phylogeny; Gene-culture co-evolution; Adaptation; Maternal mortality; Natural selection

\section{A phylogenetic approach to testing evolution- ary hypotheses using cross-cultural data}

Physiological data from a range of human populations living in different environments can provide valuable information for testing evolutionary hypotheses about human adaptation. The comparative method can help us determine whether variation results from selection due to environmental factors, or is possibly selected for by cultural forces (which means that gene-culture co-evolution

\footnotetext{
This paper is part of a collection of inter-disciplinary, peer-reviewed articles under the Theme: "Origin and Diversity of Human Physiological Adaptability" invited by K.H. Myburgh and the late P.W. Hochachka.

*Corresponding author. Tel.: +44-20-7679-2463; fax: +4420-7679-7728.

E-mail address: r.mace@ucl.ac.uk (R. Mace).
}

is occurring). However, a simple, statistical correlation between traits across cultures is complicated by the non-independence of cultures, as was first recognized in the 19th century by Francis Galton. The same problem applies in cross-species comparisons. Evolutionary biologists have developed phylogenetic comparative methods that can take account of the hierarchical inter-relatedness of species and test for the co-evolution of traits on phylogenetic trees. Mace and Pagel (1994) argued that similar approaches can be used to test crosscultural hypotheses. In this paper, we will outline two examples of the use of these approaches to test adaptive hypotheses explaining global variation in two physiological traits: the first is lactose digestion capacity in adults, and the second is population sex-ratio at birth. In the case of lactose 
(a)

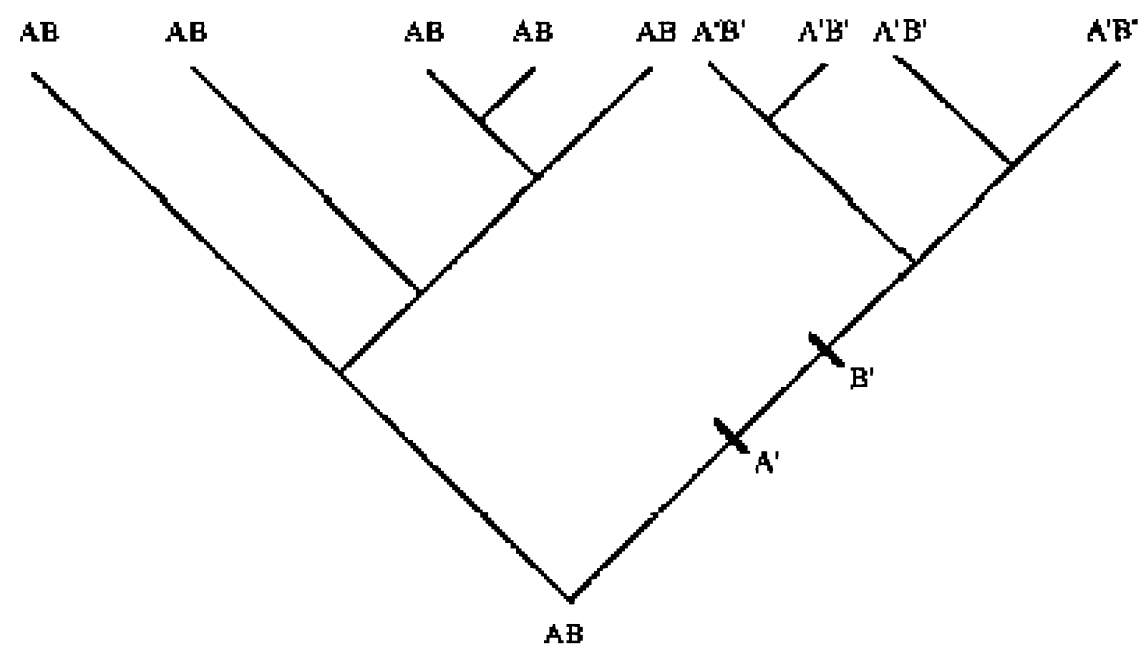

(b)

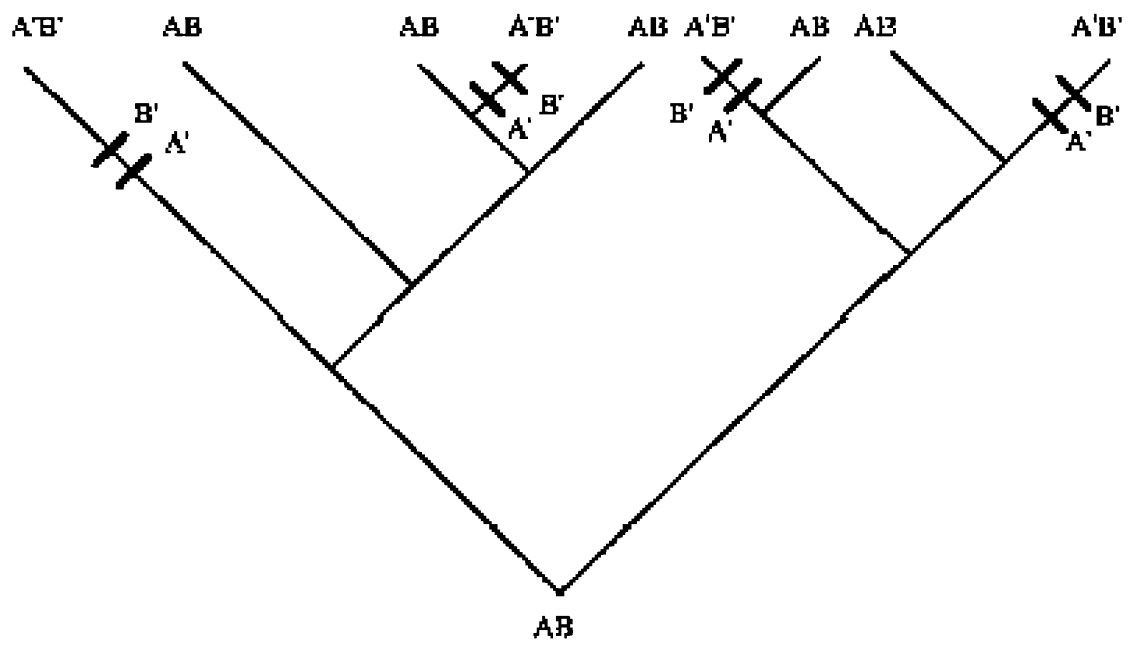

Fig. 1. Two possible scenarios in which $\mathrm{A}^{\prime}$ is evolving with $\mathrm{B}^{\prime}$. (a) A case in which four populations showing $\mathrm{A}^{\prime} \mathrm{B}^{\prime}$ and five populations showing $\mathrm{AB}$ could represent only one independent instance of $\mathrm{B}$ evolving into $\mathrm{B}^{\prime}$ after $\mathrm{A}$ had evolved into $\mathrm{A}^{\prime}$. (b) $\mathrm{A}$ case in which four populations showing $\mathrm{A}^{\prime} \mathrm{B}^{\prime}$ represents four independent instances of $\mathrm{A}$ evolving in $\mathrm{A}^{\prime}$ followed by $\mathrm{B}$ evolving into $\mathrm{B}^{\prime}$.

digestion capacity we review work we have previously published elsewhere. The study of sex ratio variation is new.

Hierarchically related units, be they species or populations, cannot be considered as statistically independent in comparative tests. Below, we report that sex ratio at birth is more male-biased in European than in African countries. This could be related to higher levels of malnutrition in Africa. A simple statistical correlation across African and European populations with some correlate of nutrition might yield a highly significant result. However, there are a large number of variables that differ between Africa and Europe. A genetic trait co-evolving with sunshine, or malaria, or polygyny, to name some arbitrary examples, could equally underlie the observed trend. Each population in Africa differing from one in Europe does not represent an independent test of the hypothesis that nutrition is influencing sex ratio, because the populations in Africa are likely to be closely related to each other, as are those in Europe. Fig. 1 illustrates how a similar correspondence between two traits across related populations could represent as little as one or as many as four independent cases of co-evolution. Clearly the latter (Fig. 1b) 
provides stronger evidence for the traits co-evolving than the former (Fig. 1a). Evolutionary biologists have developed a number of statistical approaches in which evidence for a change along the branches of a phylogenetic tree are linked to independent instances of change in the relevant trait (Felsenstein, 1985; Harvey and Pagel, 1991; Pagel, 1999).

Mace and Pagel (1994) argued that similar approaches can be used to test cross-cultural comparative hypotheses in biological and cultural anthropology. Formal methods originating in evolutionary biology can be used to construct phylogenies of cultures. Models of human population history can be inferred using genetic or linguistic data. These have been developed not only using allele frequencies to construct population genetic trees (e.g. Cavalli-Sforza et al., 1994) but also from the application of phylogenetic algorithms to cognate words in language groups (Gray and Jordan, 2000; Holden, 2002). Some debate has arisen as to whether phylogenies, a descriptive tool normally used to describe the evolutionary relationships between species, can actually be applied within species. Attempts to classify human racial groups genetically have not found discrete groups, but geographic clines of variation. Clearly some cross-cultural interbreeding occurs, such that several anthropologists have argued that a network might strictly represent population history better than a phylogenetic tree (Bateman et al., 1990; Bellwood, 2001; Moore, 1994). Trees based on language may actually suffer less from this problem of mixing, as immigrants into new communities tend to leave behind or at least fail to pass on their native language, whereas they bring their genes with them and do pass them into future generations. Hence it is possible that language trees are more tree-like than human population genetic trees. Using shared cognate words to generate trees of human cultures does produce trees that resemble historical patterns inferred from either archeological or historical sources. Both Gray and Jordan (2000) analyzing Pacific languages, and Holden (2002) analyzing Bantu languages, found that evolutionary relationships between languages suggested historical migration patterns similar to those proposed by archaeologists.

Any statistical comparison does require some explicit model of evolutionary history; not using a tree is equivalent to assuming all cultures are equally related, which we know is wrong. Networks would be very hard to estimate, and current statistical procedures cannot test for correlated evolution using networks. In the tests reported here, we do use phylogenetic trees of human cultures based on genetic similarity (Cavalli-Sforza et al., 1994). The weight of anthropological, genetic and archaeological knowledge indicates that, almost whatever the degree of cultural isolation, some populations are much more closely related to some cultures than they are to others. So whatever the shortcomings of a phylogenetic model of history, it is likely to be the best we have for our purposes here. Thus, whether the traits of interest are predominantly genetically, environmentally or culturally determined, phylogenetic regressions are appropriate.

The statistical comparative methods we use in the examples reported here are based on maximum likelihood (Pagel, 1994, 1999). ML is used to test whether the rate of change in a trait on each branch of the phylogenetic tree is influenced by the other trait on that branch of the phylogenetic tree. A model of independent evolution is fit and compared with a model in which the rate of change in one trait is influenced by the presence or absence of the other trait. If the dependent model is significantly more likely than the independent model, then this provides statistical evidence that the two traits are co-evolving. This method can be applied to discrete traits, and a similar method can be applied to continuous traits. In the case of lactose digestion capacity, the frequency distribution of levels of lactase persistence in native populations is bimodal; most un-mixed populations show either very high or low levels of lactase persistence with a trough of populations with approximately $70 \%$ of individuals showing lactose tolerance (Holden and Mace, 1997, 2002). Hence we use the method DISCRETE for discrete characters (Pagel, 1994, http://www.ams.rdg.ac.uk/ zoology/pagel/). Variation in sex-ratio at birth is a continuous trait, so in that case we used the CONTINUOUS method, which is based on generalized least squares (GLS) phylogenetic regression (Pagel, 1997, 1999, http://www.ams.rdg. ac.uk/zoology/pagel/).

\section{The evolution of lactose tolerance as an adaptation to pastoralism}

Fig. 2 shows the distribution of the frequency of high, moderate or low ability to digest lactose 


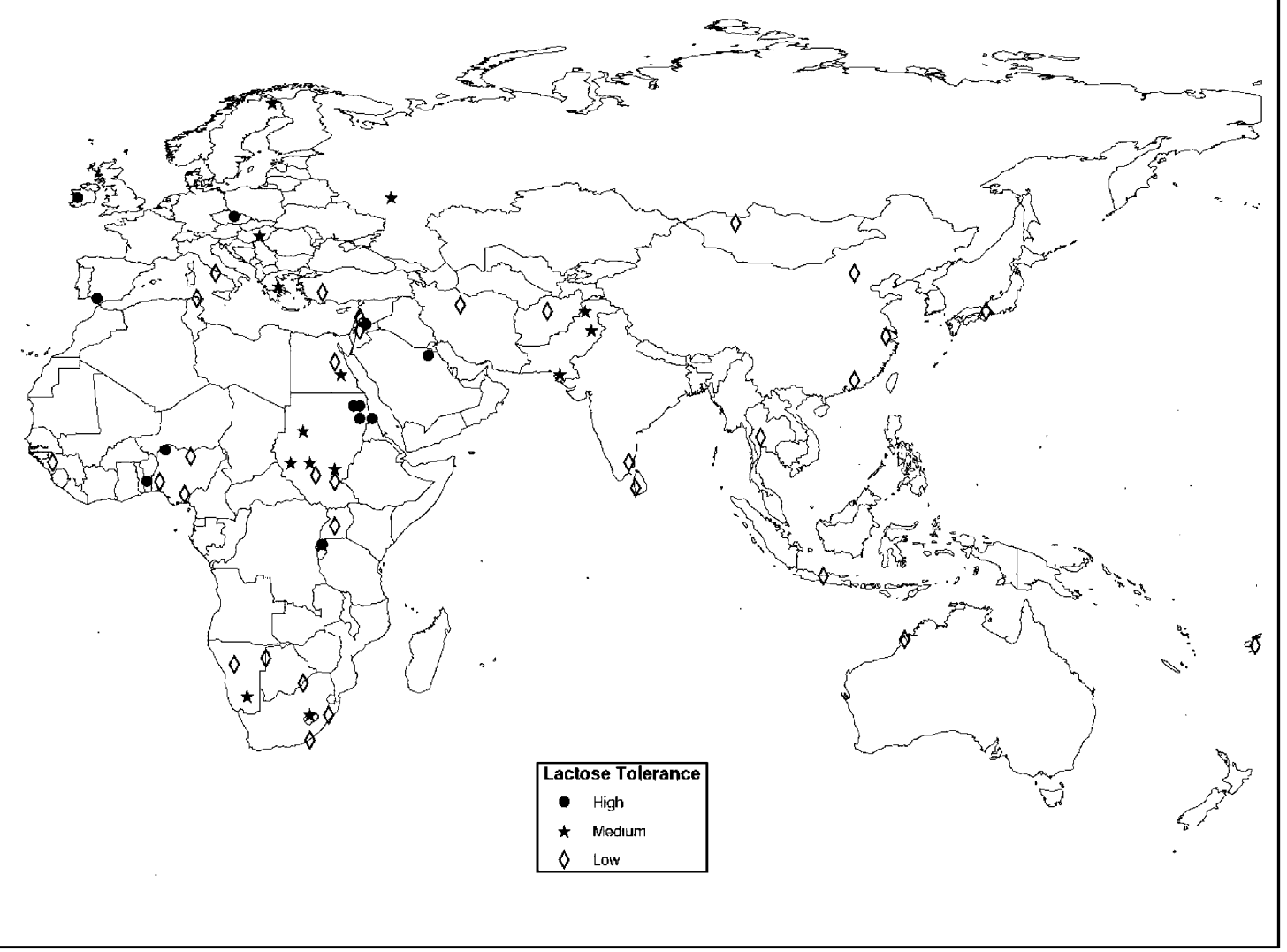

Fig. 2. Variation in levels of lactose tolerance in 56 populations where it has been measured. Six further populations that are used in the analyses are in the Americas and not shown on this map-all had low levels of lactose tolerance. Low indicates less than $30 \%$ of the population are lactose tolerant, medium indicates more than $30 \%$ but less than $70 \%$ are lactose tolerant, and high indicates at least $70 \%$ of the population are lactose tolerant. Source: Holden and Mace (1997).

in adulthood, across 56 cultures-almost all the indigenous Old World populations for which the relevant data were available. We tested the hypothesis that high levels of lactase persistence in a population was related to a history of milk-based pastoralism (Simoons, 1969, 1970, 1978). We also tested for correlations with levels of sunshine and aridity, which relate to other hypotheses for the evolution of lactase persistence (Flatz and Rotthauwe, 1973; Cook and Al-Torki, 1975; Cook, 1978). Holden and Mace (1997) constructed a phylogenetic tree mapping the relatedness of these 62 Old and New World cultures, based on the trees of genetic relatedness (six American cultures are not shown in Fig. 2-all showed low lactose tolerance) of Cavalli-Sforza et al. (1994). We compared the similarity in the measure of these traits with both geographic nearest neighbor and phylogenetic nearest neighbor. In the case of both dependence on pastoralism and lactase persistence, we found, using partial correlations, that there was a highly significant correlation between nearest phylogenetic neighbors and no correlation between nearest geographic neighbors (Holden and Mace, 2002). This shows that our model of population history gives us a great deal of information about the evolution of these traits. It suggests they are not simply spreading between neighboring populations through either cultural or biological mixing, but that mother populations are transmitting these traits to daughter populations. Hence a phylogenetic approach to testing co-evolutionary hypotheses is not only justified but essential if type 1 errors are to be avoided.

The application of the method of Pagel (1994) for detecting correlated evolution in discrete traits revealed that lactase persistence does co-evolve with pastoralism, and not with aridity or solar radiation. Furthermore, the model is able to estimate transition rates between various states, and 
estimate the most likely direction of evolutionary change. We were able to show that populations that had evolved pastoralism were very likely to evolve lactase persistence. If this particular transition was not allowed in the model, then the overall goodness of fit of the model was significantly reduced. The likelihood of populations without cattle evolving lactase persistence was low, as was the likelihood of lactase persistent populations adopting cattle. Thus by far the most likely direction of evolutionary change was that populations without lactose tolerance adopted cattle and drank the milk, and then evolved lactose tolerance. We have provided both correlational and directional evidence that supports this hypothesis.

\section{Global variation in sex ratio at birth}

Fig. 3 shows the global variation in sex ratio at birth. The data are taken from the American government CIA factbook archive [www.usa.gov. cia/factbook/15.02.2002], which is based on data compiled by numerous sources. ${ }^{1}$ Whilst few populations deviate greatly from 50:50, there is clear geographical variation in the small deviations observed. It is notable that sex ratios at birth in African countries are lower (less male-biased) than those in most of the rest of the world, and sex ratios at birth in Europe and much of Asia are high (more male-biased). In some extreme cases, specifically China and South Korea, it is likely that sex-specific abortion is so widespread that quite large deviations in sex ratio are being generated artificially (e.g. Tuljapurkar et al., 1995) and we have not included these two countries in our analyses. This artificial bias is due to widespread and accessible advanced medical facilities combined with a strong cultural preference for

\footnotetext{
1 'Information is provided by Antarctic Information Program (National Science Foundation), Bureau of the Census (Department of Commerce), Bureau of Labour Statistics (Department of Labour), Central Intelligence Agency, Council of Managers of National Antarctic Programs, Defence Intelligence Agency (Department of Defence), Department of State, Fish and Wildlife Service (Department of the Interior), Maritime Administration (Department of Transportation), National Imagery and Mapping Agency (Department of Defence), Naval Facilities Engineering Command (Department of Defence), Office of Insular Affairs (Department of the Interior), Office of Naval Intelligence (Department of Defence), US Board on Geographic Names (Department of the Interior), US Transportation Command (Department of Defence), and other public and private sources'. http://www.cia.gov/cia/ publications/factbook/index.html.
}

males. But in most of the world's population where preferences are strong such technology is not available, the observed variation is likely to largely reflect natural variation in sex ratio at birth (Fig. 3).

There is a large literature on the evolution of sex ratio. Darwinian theory predicts that the sex ratio of reproductive adults will be close to 50:50 if the costs of producing males and females are similar. Because every member of any sexually reproducing population has one mother and one father, the average reproductive success of males and females is always equal. If one sex becomes more numerous than the other, then a parent producing offspring of the rarer sex will leave more grandchildren. Hence strong selection pulls sex ratio back towards 50:50, as is usually observed in sexually reproducing species. Crucially, however, Fisher (1930) noted that it is net parental investment in boys and girls that should not differ so if one sex is more costly to produce than the other, then the more costly sex will be under-represented in proportion to that additional cost. In humans, male babies are subject to slightly higher neonatal and infant mortality than are female babies (Wells, 2000). Hence the average cost of raising a boy is lower than that of a girl, because the average number of years of parental investment given to boys is lower (as more of them receive almost no investment postpartum due to their early death). This is thought to underlie the slight male-bias in sex ratio at birth almost universally observed in human populations (Fig. 3).

However, it is not only the costs of production that are relevant, but also the benefits, which, in evolutionary terms are measured as reproductive potential. Trivers and Willard (1973) showed that, if resources invested in offspring have a greater impact on the future reproductive success of males than of females, then the optimal sex ratio of offspring could be dependent on the resources available to the mother. In most polygynous species, whilst average reproductive success of males and females is equal, variation in male reproductive success is generally much larger, and large body size often provides males with an advantage in the competition for mates. A female in poor condition is likely to produce small babies that may grow into small adults. Hence she will further her long-term reproductive success by producing a female baby. Females in good condition who can 


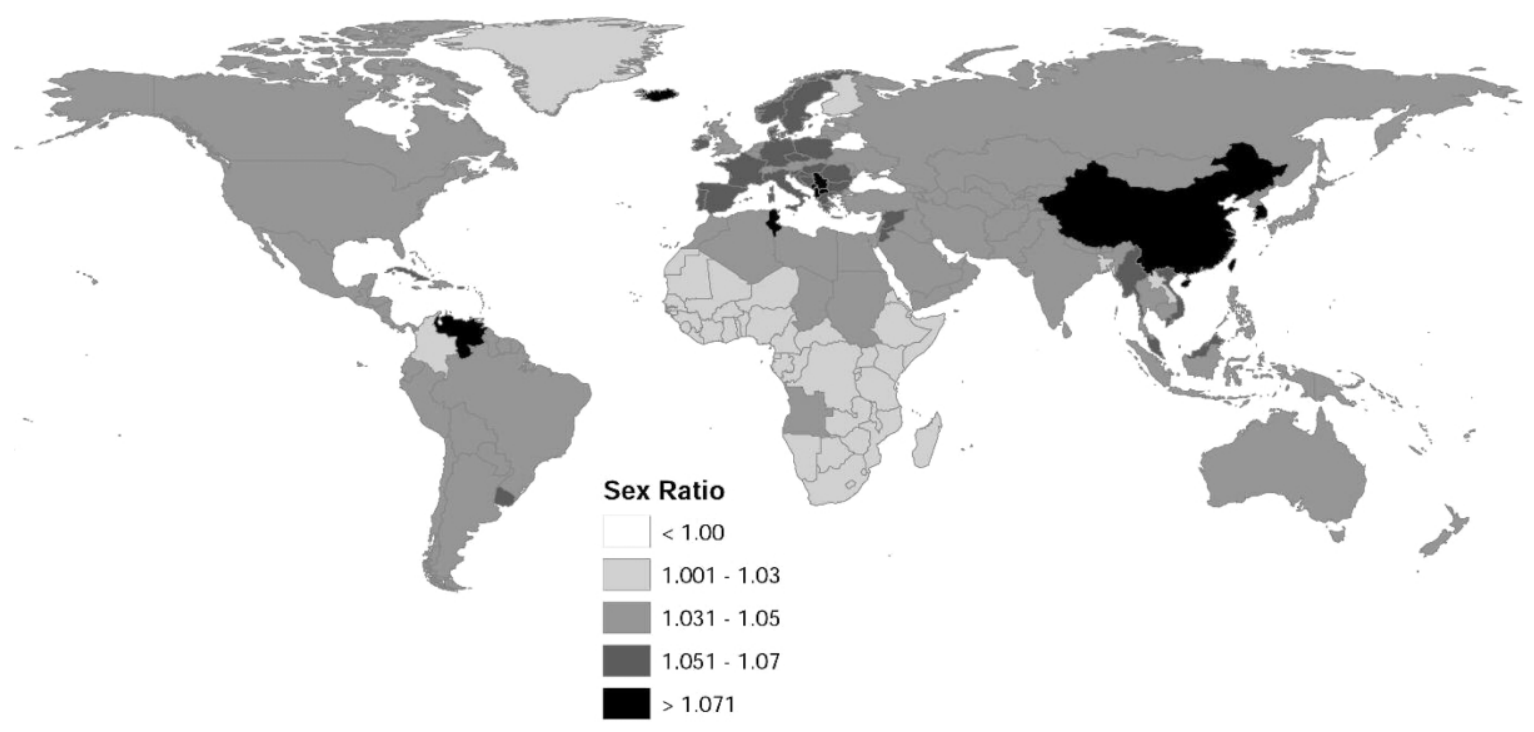

Fig. 3. Global variation in sex ratio at birth by country. Source: CIA World Factbook.

produce larger babies that will grow into tall adults, will do better by having a male. Evidence for condition-dependent sex ratio biases in line with these predictions has been shown in various populations of mammals, insects and birds (see West and Sheldon, 2002 for a review of the evidence). There is less evidence from human populations, however Gibson and Mace (in prep) have shown that in an Ethiopian population undergoing a period of food shortage, thinner women were significantly more likely to have girls than less thin women. This study is one of the few that has focused on a homogeneous group of women experiencing food shortage, which may be the conditions under which such facultative sex ratio adjustment is most evident.

It is notable from Fig. 3 that the lowest sex ratios at birth are observed in Africa, where food stress is likely to be highest. However, this cannot be readily explained by Trivers and Willard's hypothesis, as this hypothesis relates to condition dependence relative to others within the same population. Because males in Africa are not competing with males in Asia for mates, their size relative to each other is not relevant.

We believe that this bias must relate to differences in the costs and benefits to the parents of male and female children in different countries. Important costs could be biological, cultural or economic, and examining hypotheses relating to all these costs is beyond the scope of this paper.
Here we will seek evidence that biological costs are important. There is some evidence that male children are more prone to starvation that female children (Widdowson, 1976; Wells, 2000). One possibility is that regions of higher sex ratio at birth are those where male mortality in infancy and childhood is high. We will test this by examining how the sex ratio at birth is related to the sex ratio in later childhood.

Alternatively, lower sex ratios may be the result of male births imposing higher costs in terms of the mother's health and future reproductive success than do female births. Male fetuses grow faster in the womb (Marsal et al., 1996), male babies are heavier at birth (Loos et al., 2001) and are associated with a longer time elapsing for mothers' to produce their next child (Mace and Sear, 1997). It is therefore possible that the costs to the mothers health and future reproductive success of male births is larger than for female births, and that this cost will be most significant in populations where fertility is high and mortality rates (both for children and adults) are high. Below we will test for correlation between sex ratio at birth and other demographic parameters that might indicate the reproductive stress that women are under. All the country-level demographic data used are taken from the same demographic data sources as the sex ratio data, except for maternal mortality ratio, which is taken from WHO (1995). 


\section{A phylogenetic comparative analysis of sex ratio, fertility and mortality across human populations}

Here we present a cross-country comparative analysis of sex ratio at birth, where we seek to test the hypothesis that it correlates with other demographic variables, including sex ratio at higher ages, fertility and mortality levels in the general population. The distribution of the relevant traits across continents is clearly non-random, confirming the need for a method controlling for the nonindependence of cultures. We believe that sex ratio at birth is ultimately under genetic control, and we will use a phylogenetic comparative method based on a gene-based tree of human populations. In this analysis we have restricted our analysis to the Old World. New World countries (the Americas, Australia, New Zealand) currently have populations of mixed origin, and cannot therefore be informatively placed on phylogenetic trees. It is also notable that these countries appear to have more uniform sex ratios at birth near the world average of approximately 1.05 males/females, which is what we would expect of mixed populations, if this trait is under genetic control. The genetics of sex ratio is less well understood than that of lactase persistence, and neither can small scale variation be inferred from small samples as it can in the case of lactase persistence (due to the need for huge samples to avoid error), so it is not possible in this case to say whether related populations have sex ratios closer to their phylogenetic or geographic nearest neighbor. However, as stated earlier, we know that a star model for human population diversification does not accurately explain human history, and that alone requires an historical model of the relationships between cultures to be taken into account in our statistical methods. Hence phylogenetic methods are appropriate.

After selecting Old World countries for which the relevant data were available from our source, we identified the major ethnic groups of these countries and included only those where the major ethnic group could be identified on the genetic trees constructed by Cavalli-Sforza et al. (1994). Cavalli-Sforza et al. (1994) contains a worldwide genetic tree based on allele frequencies as well as separate, more detailed phylogenies for major world regions (Europe, Africa, West Asia, etc.). We matched each country's largest ethnic group to Cavalli-Sforza et al.'s populations in each of these smaller regional trees, and then constructed the overall phylogeny according to the backbone provided by the worldwide tree. The resulting tree had all branch lengths set equal to one. Countries with populations of less that 1 million were then excluded, as demographic data, especially on sex ratio at birth, might have been subject to estimation error. This left 74 countries, which are related according the tree shown in Fig. 4.

We used the phylogenetic method CONTINUOUS of Pagel (1999) for detecting correlated evolution in continuous traits, to see whether sex ratio at birth is correlated with sex ratio in childhood or adulthood, or with fertility or mortality rates.

Table 1 summarizes our results. The magnitude of the likelihood ratio (LR) is a measure of how strongly correlated the two measures are, controlling for shared phylogenetic history. $\lambda$ (lamda) is a scaling parameter that assumes a Brownian motion model of evolution and corrects tree branch length accordingly (which tends to improve the fit of the model) (Pagel, 1999, Continuous manual http://www.ams.rdg.ac.uk/zoology/pagel/). $\quad r$ indicates the correlation coefficient.

\subsection{Population sex ratio at birth and its correla- tion with sex ratio in children and adults}

There is a very strong correlation between sex ratio at birth and the sex ratio of children between the ages of 0 and 14. Sex biases at birth continue into childhood. Thus variance in sex ratios at birth across countries do not appear to be associated with variance in male infant or childhood mortality.

There is no correlation between sex ratio at birth and that in adulthood. This suggests the birth sex ratios are not responses to biases in adult sex ratio. Sex ratios at birth are not predicted to take any account of systematic sex differences in mortality rates after the period of parental investment (i.e. in adulthood). For example, even in populations where many adult males might die in warfare, those that survive are predicted to have very high reproductive success, so such effects are not predicted to bias sex ratio systematically. However, if the operational sex ratio (ratio of breeding males to breeding females) were to deviate due to some chance environmental fluctuation, then the rarer sex is at an advantage, so sex ratio could be selected to adjust accordingly. The measure used here is the sex ratio of 15-65-year olds. Although 


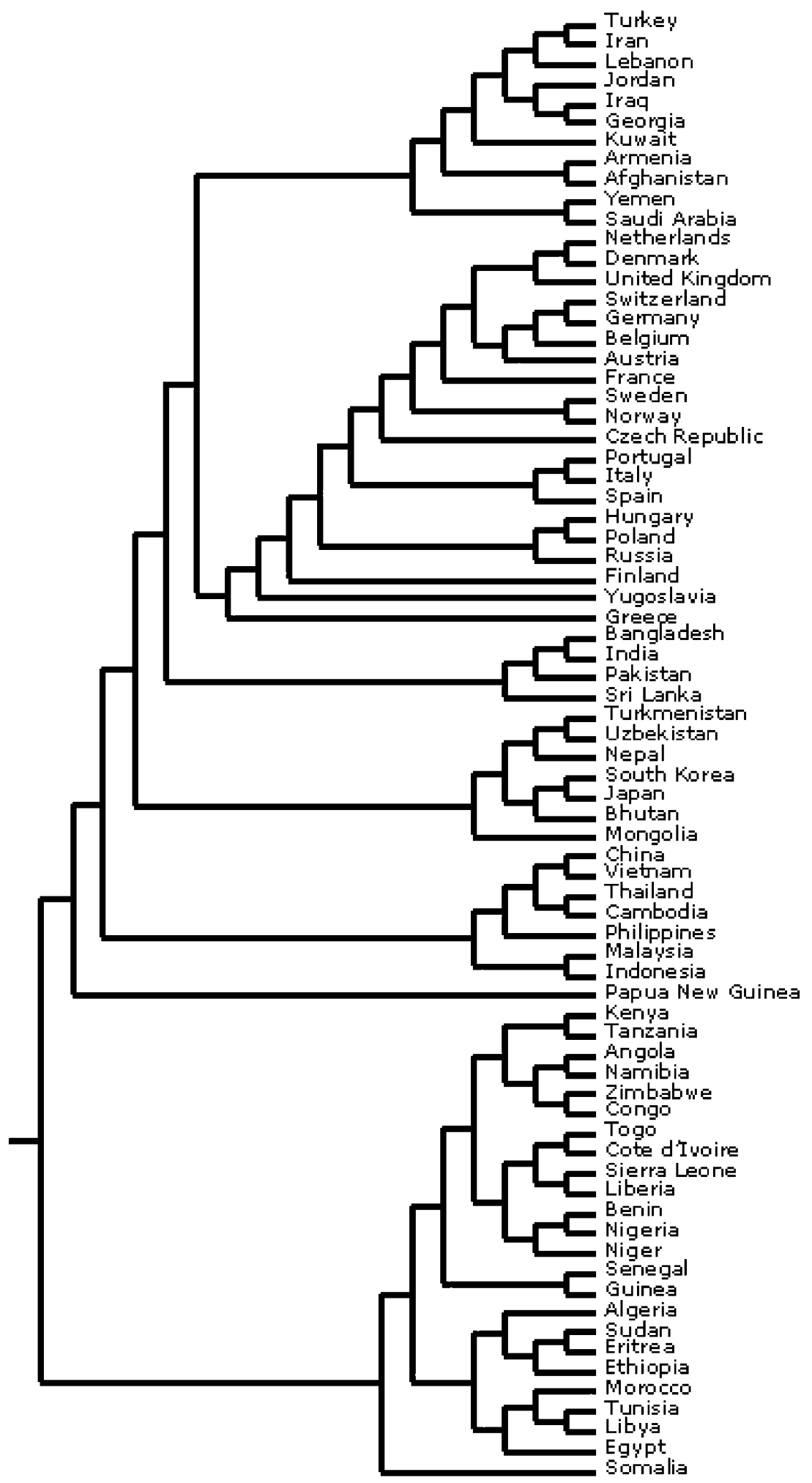

Fig. 4. Phylogenetic tree of the dominant cultures in 74 countries, adapted from Cavalli-Sforza et al. (1994). 
Table 1

Correlates of sex ratio at birth, using CONTINUOUS

\begin{tabular}{lrllcl}
\hline $\begin{array}{l}\text { Variables } \\
\text { correlated }\end{array}$ & \multicolumn{1}{l}{ LR } & $P$ & $\lambda$ & $r$ (given $\lambda)$ & \\
\hline $\mathrm{SRB} \times \mathrm{SRC}$ & 24.21 & 0.000 & 0.69 & 0.70 & $* * *$ \\
$\mathrm{SRB} \times \mathrm{SRA}$ & 0.45 & 0.341 & 0.59 & 0.11 & \\
$\mathrm{SRB} \times \ln \mathrm{nFR}$ & 3.33 & 0.010 & 0.73 & -0.30 & $* *$ \\
$\mathrm{SRB} \times \operatorname{lnIMR}$ & 1.99 & 0.045 & 0.79 & -0.23 & $*$ \\
$\mathrm{LnTFR} \times \operatorname{lnIMR}$ & 20.42 & 0.000 & 0.70 & 0.66 & $* * *$ \\
$\mathrm{SRB} \times \operatorname{lnMMR}$ & 2.60 & 0.023 & 0.70 & -0.26 & $*$ \\
\hline
\end{tabular}

$\mathrm{SRB}$, sex ratio at birth; SRC, sex ratio in children between 0 and 14; SRA, sex ratio in adults between 15 and 65 ; lnTFR, natural $\log$ of the total fertility rate; $\operatorname{lnIMR}$, natural $\log$ of the mortality rate of those under 1 year of age; lnMMR, natural $\log$ of the maternal mortality ratio (mothers death at or around childbirth per 100000 births). LR, likelihood ratio; $\lambda$ is a scaling parameter; $r$ (given $\lambda$ ) is the regression coefficient when the scaling parameter is applied (see Pagel M. Manual for CONTINUOUS http://www.ams.rdg.ac.uk/zoology/pagel/).

a proportion of the females included in this measure would be post-reproductive, this is close to the operational sex ratio of reproductive individuals; so a deviation from 50:50 in this measure probably indicates that the rarer sex should have higher reproductive success. If sex ratio at birth is influenced by that bias, then one might predict a negative correlation between sex ratio at birth and that in adulthood, however this is not seen. There is little evidence therefore that sex ratios at birth are adaptive responses to sex-biased mortality either in infancy and childhood or in adulthood.

\subsection{Sex ratio at birth and reproductive rate}

There is a significant negative correlation between sex ratio at birth and both fertility rates and infant mortality rates. The total fertility rate and the infant mortality rate of a country are very strongly correlated with each other, so it is hard to assess the independent effects of fertility or mortality. However, the correlation with fertility rate is stronger. Hence it appears that in countries where mothers give birth to many children, they are more likely to have girls. There is also a significant negative correlation with the risk per birth of death in childbirth. This further indicates that sex ratios are lowest in those countries where women's health is most endangered by their high fertility. If the physiological burden of producing males and females differ, it may be in high fertility populations that these cost differentials are greatest. High fertility may increase the health risk of pregnancy disproportionately for mothers of boys, due to their faster growth and larger size. Also, the net costs of raising girls may be lower in high fertility societies due to girls defraying their costs by helping their mothers with domestic and child-rearing duties (in ways that boys rarely do). Helle et al. (2002) found in a high fertility, historical population that mothers with a large number of daughters had longer lifespans compared to those with a large number of sons, suggesting that daughters were defraying the costs of child-rearing which had long-lasting health benefits for the mother. Our results suggest that such an asymmetry in the costs of producing and raising each sex could be selecting for lower sex ratios in high fertility populations.

\section{Discussion}

Global data on biological variation in human populations is potentially informative for testing adaptive hypotheses about the evolution of such diversity, but statistical analyses must take into account population history. We have shown here two examples of how phylogenetic comparative methods have been used to establish correlation between biological and cultural traits that vary between populations, controlling for the non-independence of cultures. In the case of lactose tolerance, there is very local diversity rather than global patterns, and the evidence for a correlation between lactose tolerance and a history of pastoralism is very strong.

In the case of variation in sex ratio at birth, we highlight variation in a trait that shows large continental variation. In this case, a phylogenetic approach is especially important if type 1 errors are to be avoided. We identify the physiological costs associated with high fertility as important correlates of low sex ratios at birth. However, there is still the possibility with phylogenetic regressions, as with any regression, that some third variable may be influencing these effects. The possibility that cultural variables, such as marriage costs, could be influencing sex ratio is an area that is important to investigate further, as Africa differs from much of Asia and Europe in this respect.

The presence of a correlation in line with an adaptive hypothesis does not necessarily inform us of whether adaptation is achieved through genetic differences between populations, or simply phenotypic plasticity i.e. a differential response to 
different environmental circumstances. Both are important to human biological adaptation. However, in the case of lactose tolerance, we know we are dealing with a simple polymorphism. In the case of sex ratio at birth, the evidence is less clear, however, it is notable that black Americans, living in the USA, have a sex ratio at birth of 1.03 , similar to that of most African populations, compared to 1.05 for white Americans (Martin et al., 2002). It has long been appreciated that population history and migration influence the biological make-up of a population. Human migration is both recent and ancient. The methods reviewed here enable us to untangle the effects of population history from those of natural selection or even cultural selection that generates this biological diversity in situ.

\section{Acknowledgments}

This research has been funded by the Wellcome Trust and the British Council. We thank Jennifer Eardley for drawing global variation in sex ratios to our attention.

\section{References}

Bateman, R., Goddard, I., O’Grady, R., et al., 1990. Speaking of forked tongues; the feasibility of reconciling human phylogeny and the history of language. Curr. Anthropol. 31, $1-13$.

Bellwood, P., 2001. Early agriculturalist population diasporas? Farming, languages, and genes. Annu. Rev. Anthropol. 30, 181-207.

Cavalli-Sforza, L.L., Menozzi, P., Piazza, A., 1994. The History and Geography of Human Genes. Princeton University Press, Princeton, NJ.

Cook, G.C., 1978. Did persistence of intestinal lactase into adult life originate on the Arabian peninsular? Man 13, 418-427.

Cook, G.C., Al-Torki, M.T., 1975. High intestinal lactase concentrations in adult Arabs in Saudi Arabia. Br. Med. J. $3,135-136$.

Felsenstein, J., 1985. Phylogenies and the comparative method. Am. Nat. 125, 1-15.

Fisher, R.A., 1930. The Genetical Theory of Natural Selection. Oxford University Press, Oxford.

Flatz, G., Rotthauwe, H.W., 1973. Lactose nutrition and natural selection. Lancet 2, 76-77.

Gray, R., Jordan, F., 2000. Language trees support the expresstrain sequence of Austronesian expansion. Nature 405, 1052-1055.

Harvey, P., Pagel, M.D., 1991. The Comparative Method in Evolutionary Biology. Oxford University Press, New York.

Helle, S., Lumaa, V., Jokela, J., 2002. Sons reduced maternal longevity in pre-industrial humans. Science 296, 1085.
Holden, C.J., 2002. Bantu language trees reflect the spread of farming across sub-Saharan Africa: a maximum-parsimony analysis. Proc. R. Soc. Lond. Ser. B 269, 793-799.

Holden, C., Mace, R., 1997. Phylogenetic analysis of the evolution of lactose digestion in adults. Human Biology 69, 605-628.

Holden, C., Mace, R., 2002. Pastoralism and the evolution of lactase persistence. In: Leonard, W.R., Crawford, M.H. (Eds.), Human Biology of Pastoral Populations. Cambridge University Press, Cambridge, UK, pp. 280-307.

Loos, R.J.F., Derom, C., Eekels, R., Derom, R., Vlietinck, R., 2001. Length of gestation and birthweight in dizygotic twins. Lancet 358, 560-561.

Mace, R., Pagel, M., 1994. The comparative method in anthropology. Curr. Anthropol. 35, 549-564.

Mace, R., Sear, R., 1997. The birth interval and the sex of children in a traditional African population: an evolutionary analysis. J. Biosoc. Sci. 29, 499-507.

Martin, J.A., Hamilton, B.E., Ventura, S.J., Menacker, F., Park, M.M., 2002. Births: Final Data for 2000. US National Vital Statistics Report 50, 1-104.

Marsal, K., Persson, P.H., Larsen, T., Lilja, H., Selbing, A., Sultan, B., 1996. Intrauterine growth curves based on ultrasonically estimate foetal weights. Acta Paediatr. 85, 843-848.

Moore, J.H., 1994. Putting anthropology back together again: an ethnographic critique of cladisitc theory. Am. Anthropol. 96, 925-948.

Pagel, M., 1994. Detecting correlated evolution on phylogenies: a general method for the comparative analysis of discrete characters. Proc. R. Soc. Lond. Ser. B 255, 37-45.

Pagel, M., 1997. Inferring evolutionary processes from phylogenies. Zool. Scr. 26, 331-348.

Pagel, M., 1999. Inferring the historical patterns of biological evolution. Nature 401, 877-884.

Simoons, F.J., 1969. Primary adult lactose intolerance and the milking habit: a problem in biological and cultural interrelations. I: review of the medical research. Am. J. Dig. Dis. 14, 819-836.

Simoons, F.J., 1970. Primary adult lactose intolerance and the milking habit: a problem in biological and cultural interrelations. II: a culture historical hypothesis. Am. J. Dig. Dis. $15,695-710$.

Simoons, F.J., 1978. The geographic hypothesis and lactose malabsorption: a weighing of the evidence. Am. J. Dig. Dis. 23, 963-980.

Trivers, R., Willard, D.E., 1973. Natural selection of parental ability to vary the sex ratio of offspring. Science 179 , 90-92.

Tuljapurkar, S., Li, N., Feldman, M.W., 1995. High sex-ratios in China's future. Science 267, 874-876.

Wells, J.C.K., 2000. Natural selection and sex differences in morbidity and mortality in early life. J. Theor. Biol. 202, 65-76.

West, S.A., Sheldon, B.C., 2002. Constraints in the evolution of sex ratio adjustment. Science 295, 1685-1688.

Widdowson, E.M., 1976. The response of the sexes to nutritional stress. Proc. Nutr. Soc. 35, 1175-1180.

WHO, 1995. Maternal Mortality in 1995: Estimates Developed by WHO, UNICEF, UNFPA. WHO; Geneva. 